\title{
Optical Design for the Large Balloon Reflector
}

\author{
German Cortes-Medellin ${ }^{\mathrm{a}, \mathrm{b}}$, Stefan O’Dougherty ${ }^{\mathrm{c}}$, Christopher Walker ${ }^{\mathrm{c}}$, Paul F. Goldsmith ${ }^{\mathrm{d}}$, \\ Chris Groppi ${ }^{\mathrm{e}}$, Steve Smith $^{\mathrm{f}}$, and Pietro Bernasconi ${ }^{\mathrm{g}}$ \\ ${ }^{a}$ Electronics and Telecom. Enginering Dep., University of Antioquia, Medellin, Colombia

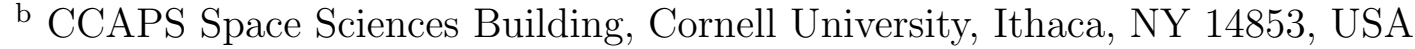 \\ ${ }^{\mathrm{c}}$ Astronomy Department, University of Arizona, Tucson, AZ 85719, USA \\ d Jet Propulsion Laboratory, Pasadena, CA 91109, USA

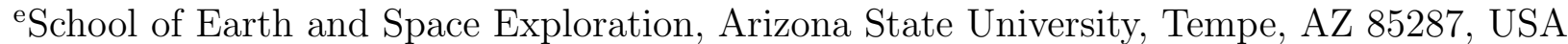 \\ fSouthwest Research Institute, San Antonio, TX 78238, USA \\ gAPL Johns Hopkings University, Laurel, MD 20723, USA
}

\begin{abstract}
We present the details of the optical design, corrector system, mechanical layout, tolerances, pointing requirements, and overall performance of the sub-millimeter wavelength Large Balloon Reflector telescope (LBR).
\end{abstract}

Keywords: Sub-mm, Stratospheric Balloon Telescope, THz Optical Design

\section{INTRODUCTION}

Balloon-borne telescopes have enabled terahertz observations of astrophysical objects which would otherwise be blocked or severely attenuated by the Earth's atmosphere. At balloon altitudes, a large portion of the submillimeter spectrum becomes accessible. Spectral lines that can be observed from airplane $(12-14 \mathrm{~km})$ altitudes with difficulty, and for which certain velocities are completely "blocked" by atmospheric absorption, become readily observable with nearly perfect transmission from balloon $(37-40 \mathrm{~km})$ altitude. One important example is the $63 \mu \mathrm{m}$ fine structure transition of neutral oxygen ([OI]). This line is an important coolant of the relatively hot layers of molecular clouds close to young, massive stars -in Photon Dominated Regions (PDRs). In addition to its biological importance, water is a key molecule for astronomy, in that it is a major coolant of dense, gravitationally bound condensations, allowing them to contract and form new stars, starting from modest temperatures of $50-100 \mathrm{~K}$. Water has been found in a wide variety of objects including such cloud cores, protostellar disks in which planetary systems are forming, as well as primitive objects in our solar system such as comets. Systematic observations of all of these will be necessary to trace the "water trail" connecting water in interstellar clouds to the Earth's water. However, objects in our solar system and most of the Milky Way cannot be observed in water lines even from the SOFIA airborne observatory because the atmosphere is completely opaque. From a balloon, however, almost the entire galaxy can be observed in a wide range of water lines, which together can yield critical information about the molecule's excitation, and thus on its abundance and cooling. For the solar system, observations of the most common isotopologue, $\mathrm{H}_{2}^{16} \mathrm{O}$, are challenging due to the small Doppler shifts, but taking advantage of the Earth's motion, many objects will be observable at the right time of the year. The rare variants, $\mathrm{H}_{2}^{18} \mathrm{O}$ and $\mathrm{HDO}$ are of particular interest for cometary studies as they may well be able to answer whether cometary impacts early in the history of the solar system were the origin of the Earth's water. The $557 \mathrm{GHz}$ ground state transition of ortho- $\mathrm{H}_{2} \mathrm{O}$ is a key objective for LBR. The unequaled angular resolution and sensitivity at the frequency of this important astronomical tracer will certainly yield spectacular new results for a wide range of objects of great astronomical interest.

Balloon borne missions such as STO (Stratospheric THz Observatory), ${ }_{1}^{1}$ BLAST (Balloon-borne Large Aperture Sub-millimeter Telescope), ${ }^{2,3}$ and BLAST-Pol ${ }^{4,5}$ have proven that hanging a telescope from a balloon is a very economical approach for terahertz astronomy compared to the cost of flying space-borne instruments. Nevertheless, one of the drawbacks is the ultimate pointing accuracy of the hanging telescope, which restricts the use of large apertures at very high frequencies.

Ground-based and Airborne Telescopes VI, edited by Helen J. Hall, Roberto Gilmozzi, Heather K. Marshall, Proc. of SPIE Vol. 9906, 99061Y · C 2016 SPIE · CCC code: 0277-786X/16/\$18 · doi: 10.1117/12.2233861 

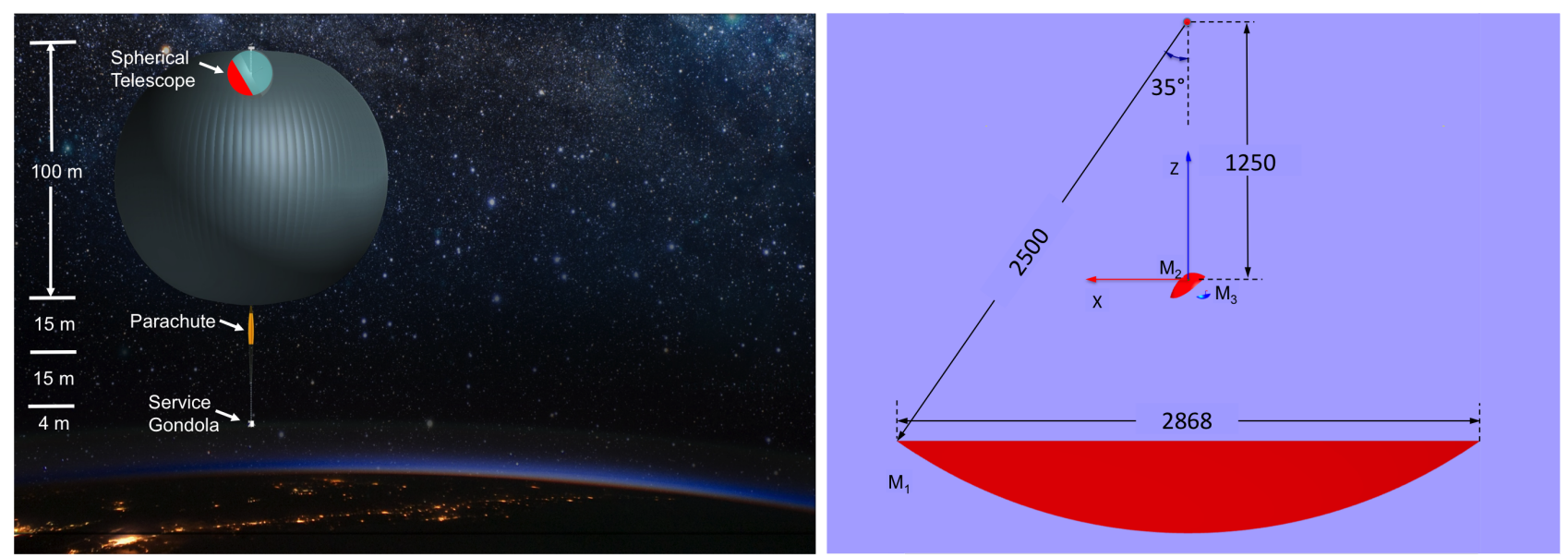

Figure 1. Left: Large Ballon Reflector Sub-mm wave telescope concept mounted inside a large stratospheric balloon. Right: Spherical cap of a $5 \mathrm{~m}$ diameter LBR balloon telescope (all dimensions in $\mathrm{mm}$ ).

A new concept, the Large Balloon Reflector (LBR) has been proposed and funded through the NASA Innovative Advanced Concepts (NIAC) program. In this approach the main reflector of the telescope is a metallized hemisphere of a smaller spherical balloon, 5 to $20 \mathrm{~m}$ in diameter, inflated inside the much larger $(100 \mathrm{~m})$ carrier stratospheric balloon. Light from space first passes through the $\sim 50 \mu \mathrm{m}$ thick polyethylene skin of the carrier balloon and then through the $\sim 25 \mu \mathrm{m}$ thick Mylar side of the inner balloon. Together these layers have $<8 \%$ absorption at the wavelengths of interest. The incoming light then encounters the aluminized, spherical, back surface of the inner balloon and is then focused into a spherical corrector optics and terahertz receiver sit in the interior of the sphere (See Fig. 1-Left). This location effectively shields the spherical telescope from external atmospheric effects that normally would influence the instrument hanging from a gondola about $30 \mathrm{~m}$ under the main balloon.

We present here the details of the optical design, corrector system, mechanical layout, tolerances, pointing requirements, and overall expected performance of a $5 \mathrm{~m}$ prototype LBR.

\section{LARGE BALLOON REFLECTOR OPTICS}

\subsection{Spherical Corrector for a $5 \mathrm{~m}$ Sub-mm Spherical Telescope}

Since LBR's main reflector (M1) is a spherical surface an spherical corrector system is needed to compensate. The corrector system is based on a scaled version of the $300 \mathrm{~m}$ Arecibo radio telescope Gregorian system. ${ }^{6}$ The corrector system is an off-set double Gregorian reflector that refocuses the incoming field at the foci of the optics eliminating the spherical aberration. Fig. 1-(Right) shows the dimensions of a $5 \mathrm{~m}$ diameter LBR telescope concept with spherical corrector in place. The spherical cap is part of a spherical sector with a vertex angle of $70^{\circ}$. This vertex angle yields an aperture of $2868 \mathrm{~mm}$ in diameter for a $5 \mathrm{~m}$ spheric reflector. This is larger than the actual effective aperture of the corrector optics in order to control spillover. The Gregorian corrector system sits at a distance of half radius from the center of the sphere. The spherical corrector system for LBR telescope consist of two highly shaped surfaces, M2 and M3, needed to correct the incoming spherical wavefront aberration as evidentiated by the caustic lines in the ray tracing shown in Fig. 2-(Left). The secondary reflector (M2) is $130 \mathrm{~mm}$ in height, by $178 \mathrm{~mm} \times 218 \mathrm{~mm}$ wide. The tertiary reflector (M3) is smaller: $29 \mathrm{~mm}$ in height by $72 \mathrm{~mm} \times 65 \mathrm{~mm}$ wide. Fig. 2-(Right) shows an oblique view of the Gregorian corrector system showing the larger wide dimensions of M2 and M3 reflectors. The incoming wavefront forms a half full angle of $60^{\circ}$ at the foci of the telescope optics.

\subsection{Performance at $557 \mathrm{GHz}$}

Fig. 3-(Left) shows the aperture field power distribution of the $5 \mathrm{~m}$ LBR telescope with the dual Greforian reflector corrector system at a frequency of $557 \mathrm{GHz}$. The illumination in the aperture is nearly uniform and elliptical 

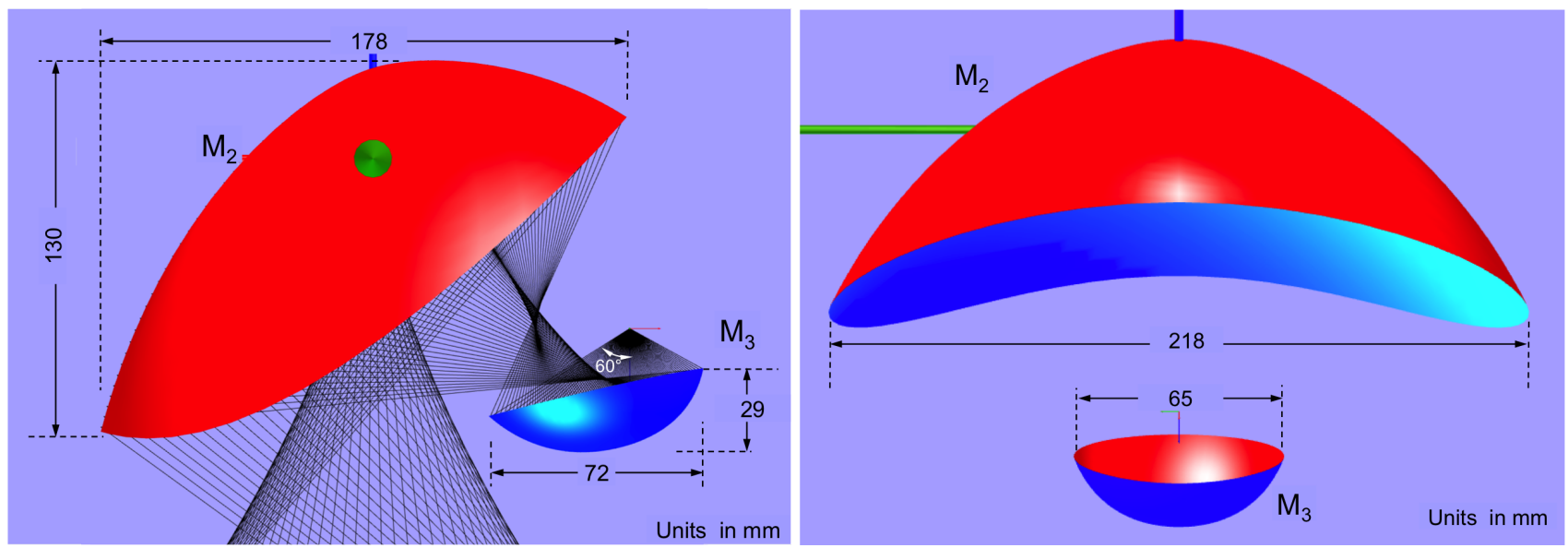

Figure 2. Left: Side view of the spherical corrector system consisting of two surfaces M2 and M3. Right: Oblique view of the corrector system. The dimensions correspond to a $5 \mathrm{~m}$ diameter LBR balloon telescope.

in shape with major and minor diameter dimensions of $2263 \mathrm{~mm} \times 1980 \mathrm{~mm}$ respectively. The figure shows the blockage produced by the corrector structure at the center. Fig. 3-(Right) shows the corresponding aperture phase distribution of the LBR telescope with corrected optics at $557 \mathrm{GHz}$ indicating a residual phase error rms of $1.2 \times 10^{-4} \lambda$ across the aperture, equivalent to a Strehl ratio of $\sim 100 \%$.
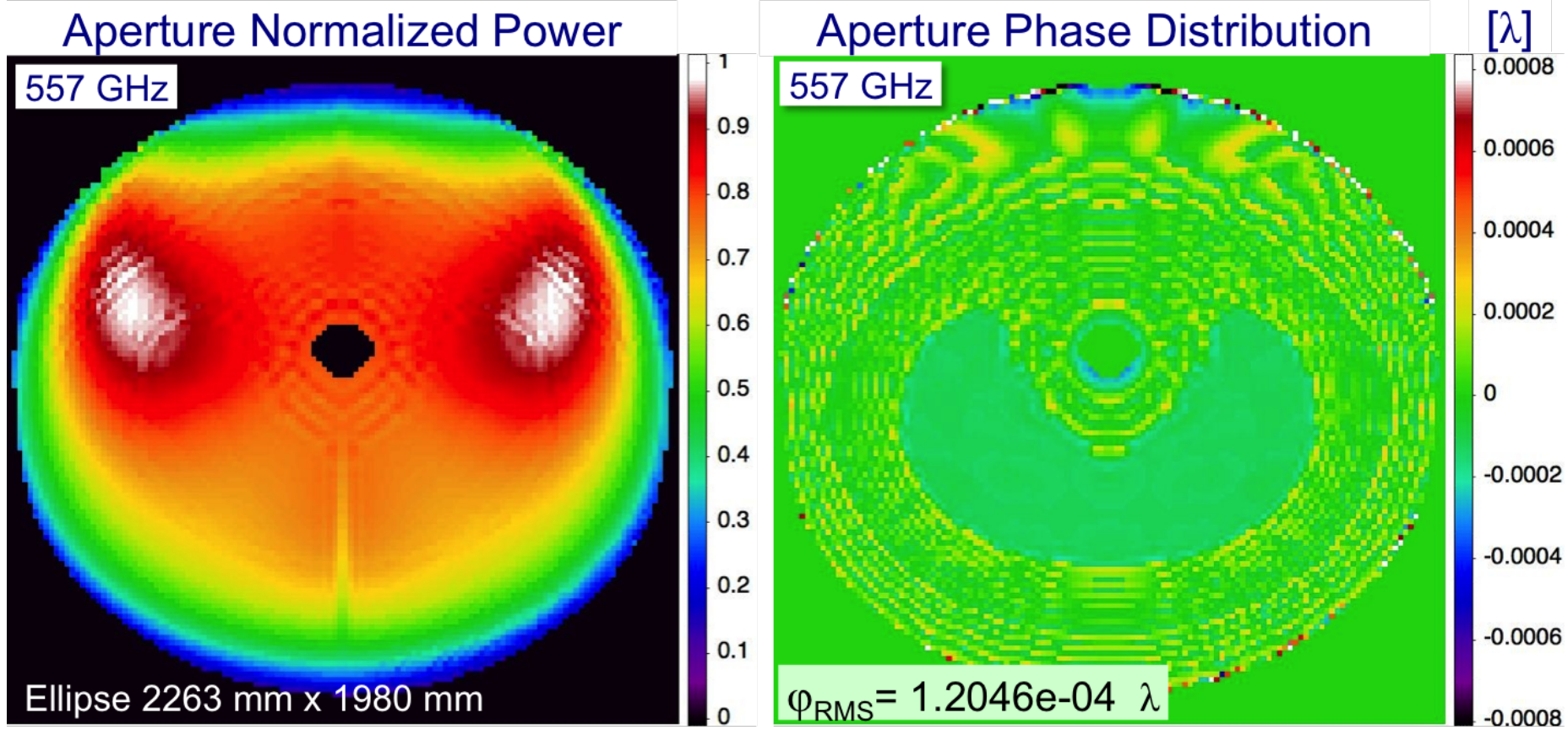

Figure 3. Left: Aperture field power distribution of the LBR telescope with the spherical corrector system at $557 \mathrm{GHz}$. The aperture is elliptical in shape with major and minor diameter dimensions of $2263 \mathrm{~mm}$ and $1980 \mathrm{~mm}$ respectively. Right: Corresponding aperture phase distribution at a frequency of $557 \mathrm{GHz}$. The residual phase errors of the corrected optics are less than $1.2 \times 10^{-4} \lambda$.

Fig. 4-(Left) shows the corresponding (linear polarization) co-polar far field radiation pattern of the 5m LBR telescope with corrected optics at a frequency of $557 \mathrm{GHz}$. The panels depict a Field of View area in the sky of $13.35 \mathrm{arcmin}^{2}$. The main beam is elliptical in shape with a FWHM of 59.5 arcsec $\times 52.4$ arsec. The nearly uniform aperture power distribution produces a sidelobe level of $-16.2 \mathrm{~dB}$. Fig. 4-(Right) shows the far field cross- 
Co-Polar Far Field Pattern

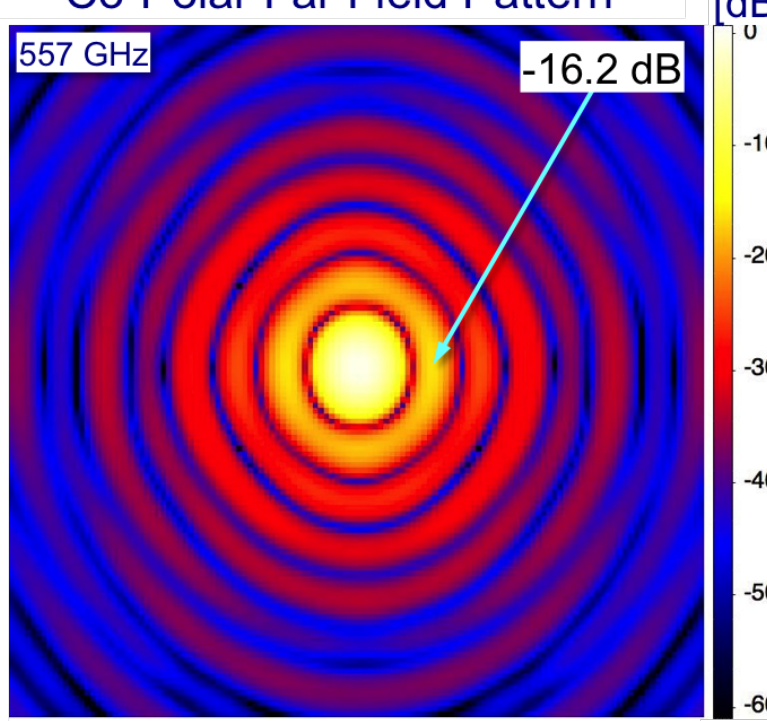

Area: 13.35 arcmin $\times 13.35$ arcmin
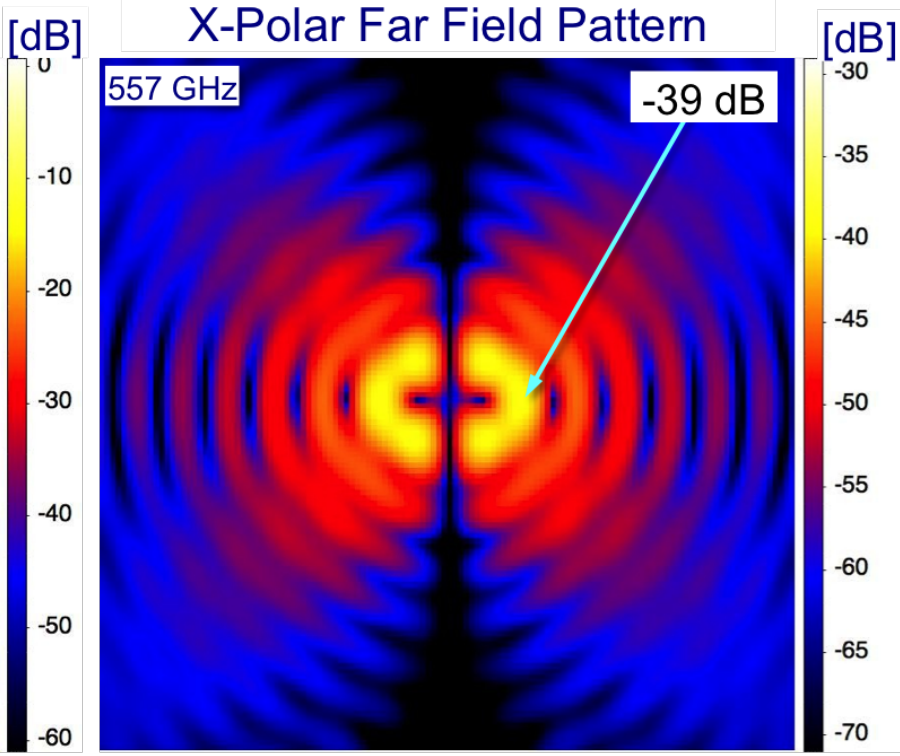

\section{HPBW: 59.5 arcsec $\times 52.4$ arcsec}

Figure 4. Left: Far field Co-polar antenna pattern of the $5 \mathrm{~m} \mathrm{LBR}$ telescope with the spherical corrector system at $557 \mathrm{GHz}$. The main beam HPBW is elliptical in shape with a size of $59.5 \operatorname{arcsec} \times 52.4$ arsec. Right: Corresponding cross-polar far field antenna pattern of the $5 \mathrm{~m}$ at $557 \mathrm{GHz}$, with peak cross-pol level of $-39 \mathrm{~dB}$.

polar radiation pattern at $557 \mathrm{GHz}$, indicating excellent cross-polarization performance, with a peak cross-pol value of $-39 \mathrm{~dB}$ across the field.

In Fig. 5 we show a series of $\phi$-cuts near the main beam of the far field radiation pattern at $\phi=0^{\circ}, 45^{\circ}$ and $90^{\circ}$ respectively. As it is evident from the cuts, the first sidelobe level presents some azimuthal variation, between $-16 \mathrm{~dB}$ to $-18 \mathrm{~dB}$. The cross-polarization performance determined by the $45^{\circ}$ diagonal pattern cut indicate a peak value of cross-polarization of $-39 \mathrm{~dB}$.

Table 1 summarizes the LBR telescope performance at $557 \mathrm{GHz}$, with a gain of $81.5 \mathrm{~dB}$, beam efficiency of $99.9 \%$, and an overall aperture efficiency of $92.4 \%$.

Table 1. Overall Telescope Performance for a 5 m LBR

\begin{tabular}{|l|c|l|}
\hline Parameter & Value & Units \\
\hline \hline Wavelength & 538 & $\mu \mathrm{m}$ \\
Frequency & 557 & $\mathrm{GHz}$ \\
Gain & 81.5 & $\mathrm{~dB}$ \\
Cross-Pol & -39.1 & $\mathrm{~dB}$ \\
FWHM & $59.2 \times 52.4$ & $\operatorname{arcsec}$ \\
Image Plate Scale x-axis & 3.6 & $\operatorname{arcmin} / \mathrm{mm}$ \\
Image Plate Scale y-axis & 2.8 & $\operatorname{arcmin} / \mathrm{mm}$ \\
Effective Aperture & 3.23 & $\mathrm{~m}^{2}$ \\
Aper. Phase rms & $1.2 \times 10^{-4}$ & $\lambda$ \\
Aper Illum. Efficiency & 96.9 & $\%$ \\
Aper Phase Efficiency & 99.9 & $\%$ \\
Spillover and Blocking Efficiency & 99.9 & $\%$ \\
Beam Efficiency & 99.9 & $\%$ \\
\hline \hline Total Antenna Efficiency & $\mathbf{9 1 . 8}$ & $\%$ \\
\hline \hline
\end{tabular}




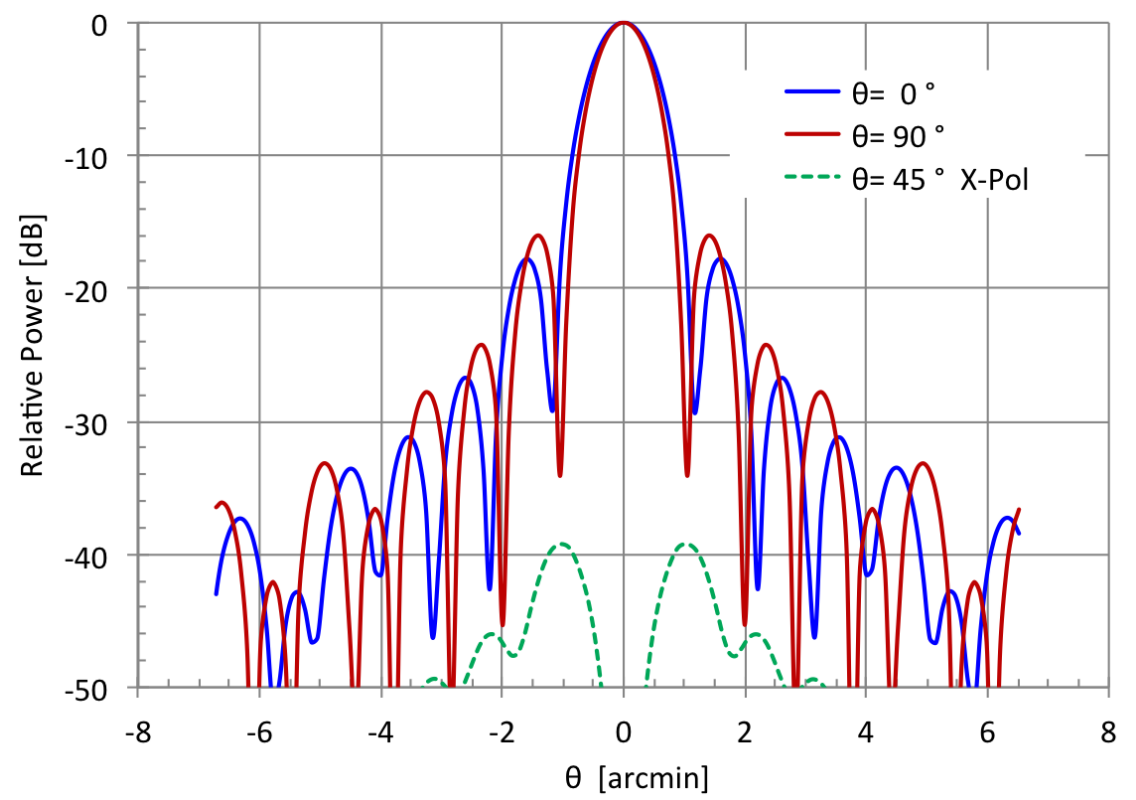

Figure 5. Cuts in the principal planes of the calculated far field radiation pattern, near the main beam, at $557 \mathrm{GHz}$ of the 5-m diameter LBR's. The cuts are at $\phi=0^{\circ}, 45^{\circ}$ and $90^{\circ}$ respectively. The figure indicates a cross-polar peak value of $-39 \mathrm{~dB}$.

\subsection{Field of View}

Fig. 6 shows the calculated characteristics of the field of view (FoV) of the $5 \mathrm{~m}$ LBR telescope with spherical correction optics. The correction optics is off-set, therefore there is a small asymmetry between the x-axis and y-axis. (See Fig. 1-Right and Fig. 2). Assuming a typical value of tolerable optical performance degradation of $-1.0 \mathrm{~dB}$ (or approximately $80 \%$ efficiency), the FoV can accommodate up to $6 \mathrm{x} 6$ beams, separated by one FHWM, (i.e., 59 arcsec at $557 \mathrm{GHz}$ ) with a power scan loss better than -1dB. The image plate scale is:

$$
\begin{aligned}
& \mathrm{IMS}_{X}=3.6 \mathrm{arcmin} / \mathrm{mm} \\
& \mathrm{IMS}_{Y}=2.8 \text { arcmin } / \mathrm{mm} \text {. }
\end{aligned}
$$

This indicates relatively small field distortion: i.e., compression along the $\mathrm{y}$-axis and stretching along the $\mathrm{x}$-axis

\section{CORRECTOR SYSTEM POSITION SENSITIVITIES}

One of the more challenging aspects of balloon born telescopes is the pointing, and the problem aggravates with aperture size in terms of wavelength.

\subsection{Feed Positioning Sensitivities}

Fig. 7 shows the calculated scanning loss with respect to feed position in the focal plane, i.e., lateral displacements, along the $\mathrm{x}$-axis and $\mathrm{y}$-axis, as well as defocusing in a direction perpendicular to the focal plane (along the local z-axis) for a $5 \mathrm{~m}$ class LBR telescope at frequency of $557 \mathrm{GHz}$. Lateral feed displacements along the y-axis in the focal plane up to $\pm 1 \mathrm{~mm}$ produce scanning losses of $-0.8 \mathrm{~dB}$. Along the $\mathrm{x}$-axis, lateral feed displacements are somewhat more restrictive, as scanning losses of $-1.0 \mathrm{~dB}$ are produced by lateral errors of $-0.8 \mathrm{~mm}$ to + $1 \mathrm{~mm}$ along the x-axis. Nevertheless, focusing errors, along the focal plane normal (z-axis), is what dominate the sensitivity error budget, i.e., in order to maintain gain variations smaller than $10 \%$, the focusing error should be less than $\pm 0.3 \mathrm{~mm}$. This implies that the feed position should be maintained within $\pm 300 \mu \mathrm{m}$, while scanning the focal plane.

Based on the optical design a point cloud data set was generated for M2 and M3 to create a solid model shown in Fig. 8 in solid Autodesk Inventor for high precision machining. The surface precision requirements 


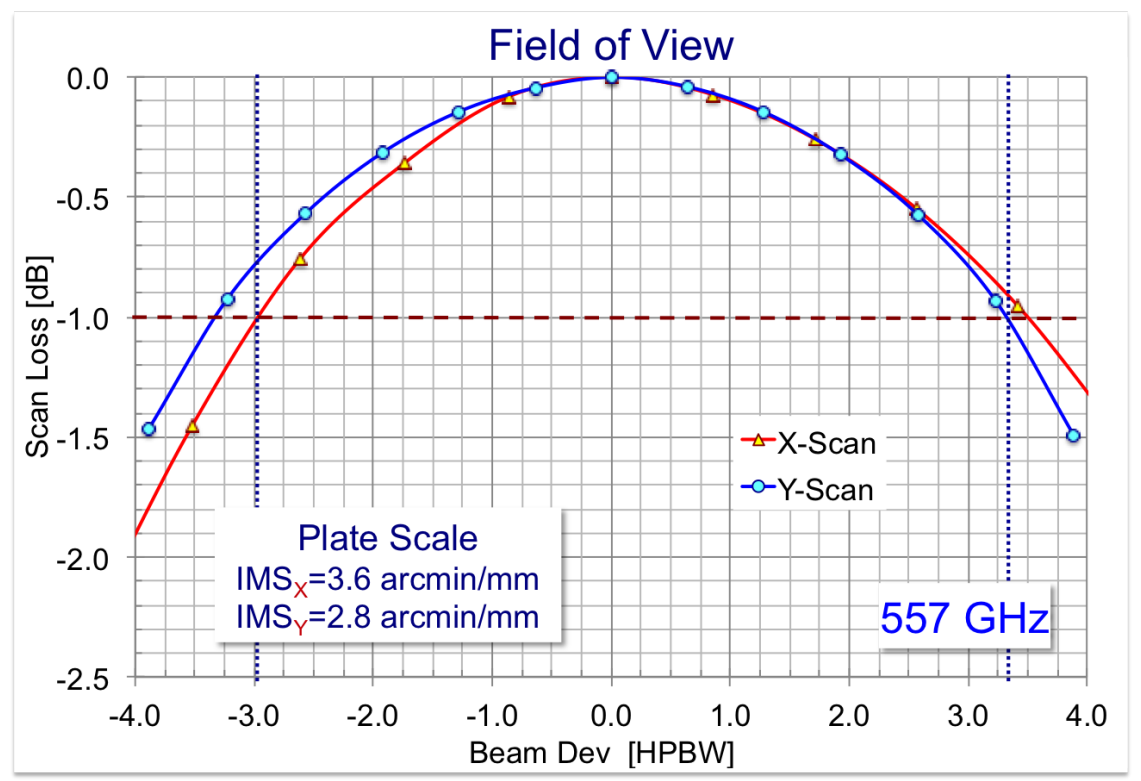

Figure 6. Field of View and plate-scale at the focus of the spherical corrector optics..

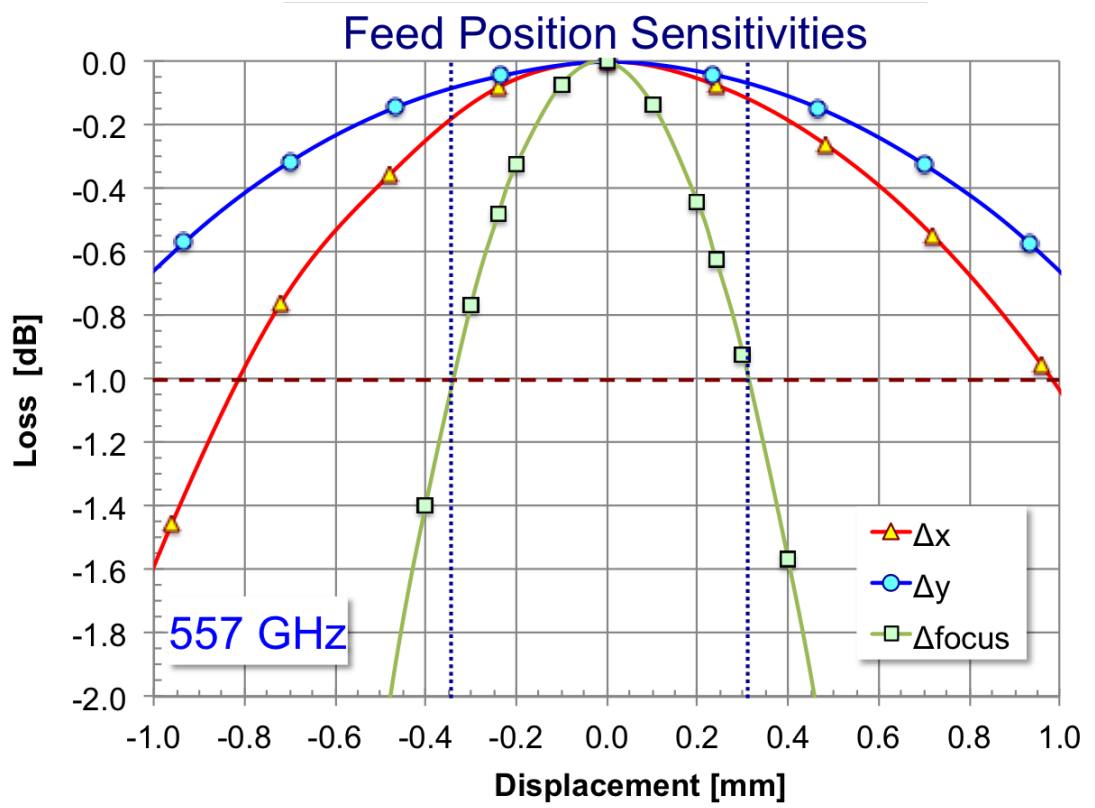

Figure 7. Calculated scanning loss as a function of Feed position in the focal plane of $5 \mathrm{~m}$ class LBR telescope with spherical corrector system at $557 \mathrm{GHz}$.

are of the order of $\pm 30 \mu \mathrm{m}$ for the overall surface rms, for good optical performance, but what dominates is the pointing requirement of $1 / 10^{\text {th }}$ of the beam, which at $557 \mathrm{GHz}$ is 6 arc sec.

\subsection{Corrector Assembly Sensitivities}

In the previous section we answered the question of how precisely we have to position the feed at the foci of the optics in order to maintain good optical performance. In this section we answer the question of how precisely we have to position the whole corrector assembly (corrector reflector optics and feed) in order to maintain good optical performance. Fig. 9 shows the calculated gain losses as a function of positioning errors of the whole corrector assembly: lateral along the $\mathrm{x}$-axis and y-axis, and along the z-axis. We see that the optics is very 


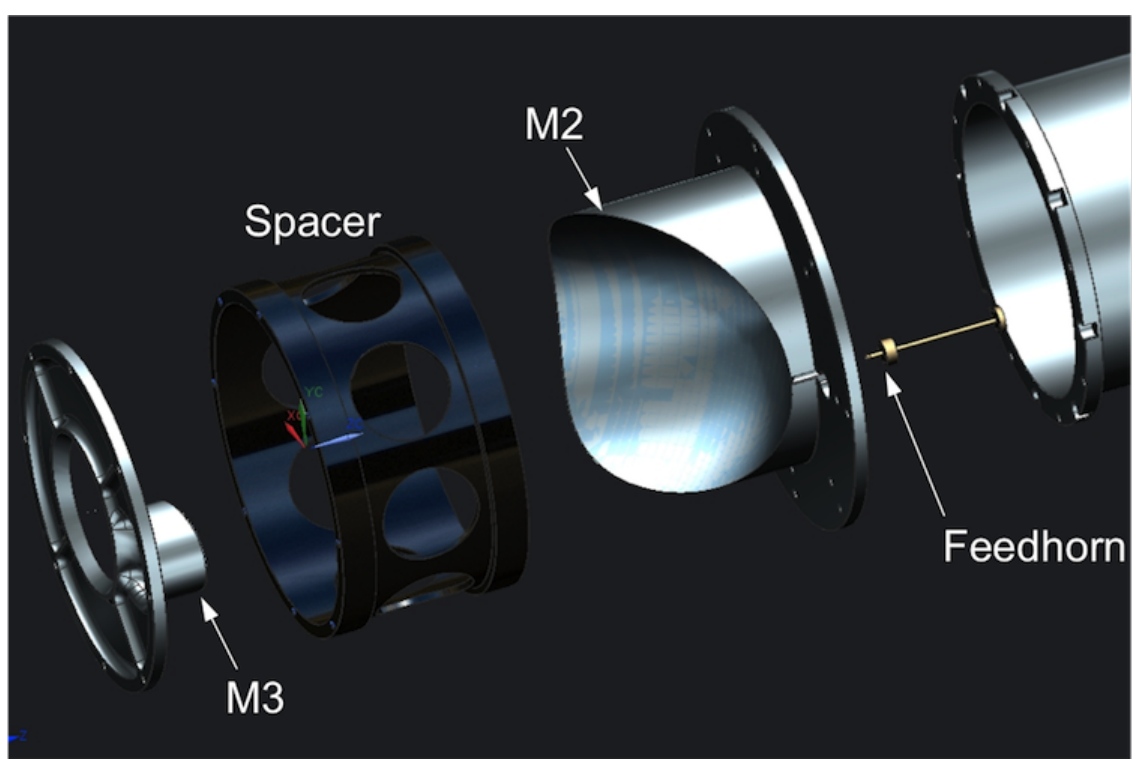

Figure 8. Details of the corrector assembly solid model (Arizona State University).

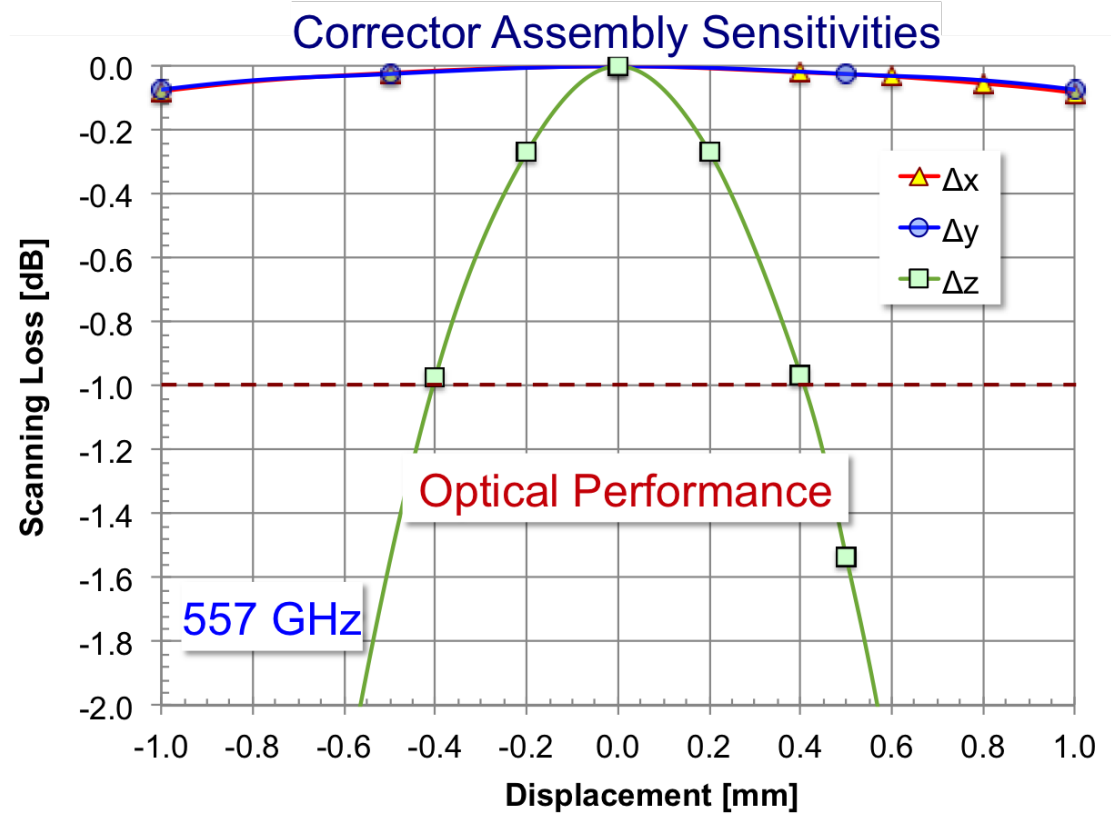

Figure 9. Calculated scanning loss a a function of corrector assembly positioning at $557 \mathrm{GHz}$.

insensitive to lateral displacements of the corrector assembly as a whole beyond $\pm 1 \mathrm{~mm}$. Nevertheless, in order to ensure good optical performance (i.e., scanning loss better than $-1.0 \mathrm{~dB}$ within the FoV) z-axis positioning errors should be kept within $\pm 400 \mu \mathrm{m}$.

\subsection{Corrector Assembly Sensitivities and Pointing}

Pointing requirements are normally $1 / 10^{\text {th }}$ of the beam or 6 arsec. The image plate scale presented in Equation 1 refers to the feed at the focal plane. For the whole corrector assembly the Beam Deviation Factors (BDF) are smaller:

$$
\begin{array}{lll}
\text { Assembly } \mathrm{BDF}_{X}=2.4 & \operatorname{arcmin} / \mathrm{mm} \\
\text { Assembly } \mathrm{BDF}_{Y}=2.3 & \operatorname{arcmin} / \mathrm{mm}
\end{array}
$$


Positioning errors have the largest impact on pointing rather than on optical performance: i.e., in order to maintain pointing within $1 / 10^{t h}$ of the beam, we have to maintain the lateral positioning of the corrector assembly within $\pm 41 \mu \mathrm{m}$. Since this is 10 times more stringent than the positioning requirement for good optical performance, therefore, if we maintain the corrector assembly within the pointing positioning requirements, it will also result in very good optical performance. The pointing of the telescope will be implemented in several stages: the first stage will be at the elevation/azimuth control of the spherical balloon attached to the apex platform of the carrier balloon, a second stage will be at an active fine positioning control of the corrector assembly, and finally by using an active secondary reflector $\left(M_{2}\right)$ that will make corrections to the aperture phase distribution, including the "saddle" gravitational effect.

\section{BALLOON DEFORMATION UNDER GRAVITY}
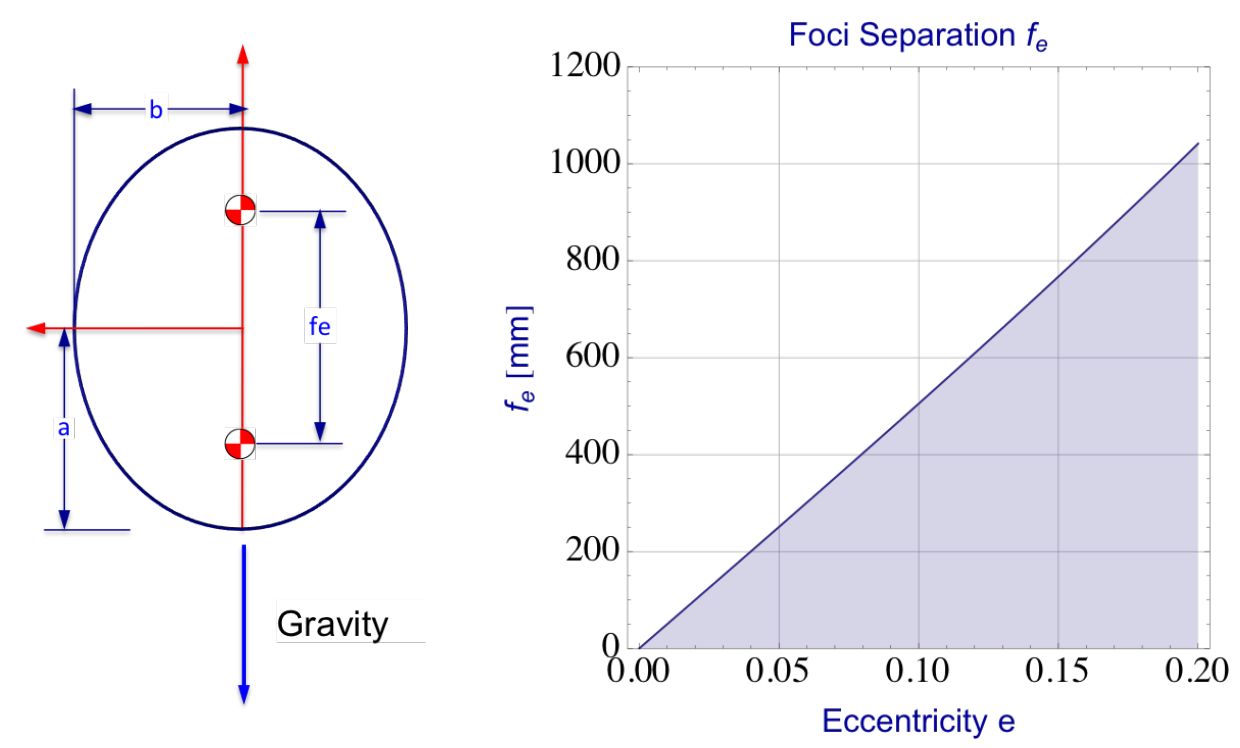

Figure 10. Left: Ballon deformation under gravity changes in first order the eccentricity. Right: Foci separation $f_{e}$ as a function of eccentricity.

We also assessed the impact in the optics of balloon deformation under gravity. We expect that the balloon will deform axially along the gravity vector into an ellipsoid (See Fig. 10-Left). At first order, gravity will increase the eccentricity of the main reflector, this will produce the separation between foci a distance $f_{e}$, i.e.,

$$
\begin{aligned}
e^{2} & =1-\left(\frac{a}{b}\right)^{2} \\
f_{e} & =\frac{2 e \mathcal{R}_{c_{e}}}{\left[1-e^{2}\right]},
\end{aligned}
$$

where $a$ and $b$ are de major and minor semi-axis, $\mathcal{R}_{c_{e}}$ is the radius of curvature at the vertex of the deformed balloon. We assumed that the vertex radius of curvature remains constant under this type of deformation and characterized the phase error in the aperture for different values of eccentricity. Fig. 10-(Right) shows the distance between the ellipsoid foci as a function of eccentricity.

Using a fixed value of radius of curvature of the balloon vertex $\mathcal{R}_{c_{e}}=2500 \mathrm{~mm}$, we varied the eccentricity of the main reflector from 0.01 to 0.2 , and evaluated the optical performance paying special attention to the aperture phase distribution. Fig. 11-(Left) shows the aperture phase distribution for an eccentricity value of $e=0.03$, which it is equivalent to a foci separation of $0.15 \mathrm{~m}$, and at a wavelength of $540 \mu \mathrm{m}$ produces s Strehl ratio value of $99.1 \%$ Fig. 11-(Right) shows the aperture phase distribution for a value of eccentricity $e=0.2$, or a foci septation of $1.05 \mathrm{~m}$. (at $\lambda=50 \mathrm{~mm}$ ). We found that the shape of the aperture phase distribution remains invariant (saddle) for all the values of eccentricity (independent of the wavelength used to assess it). This means that it could be easily corrected by calibration. 

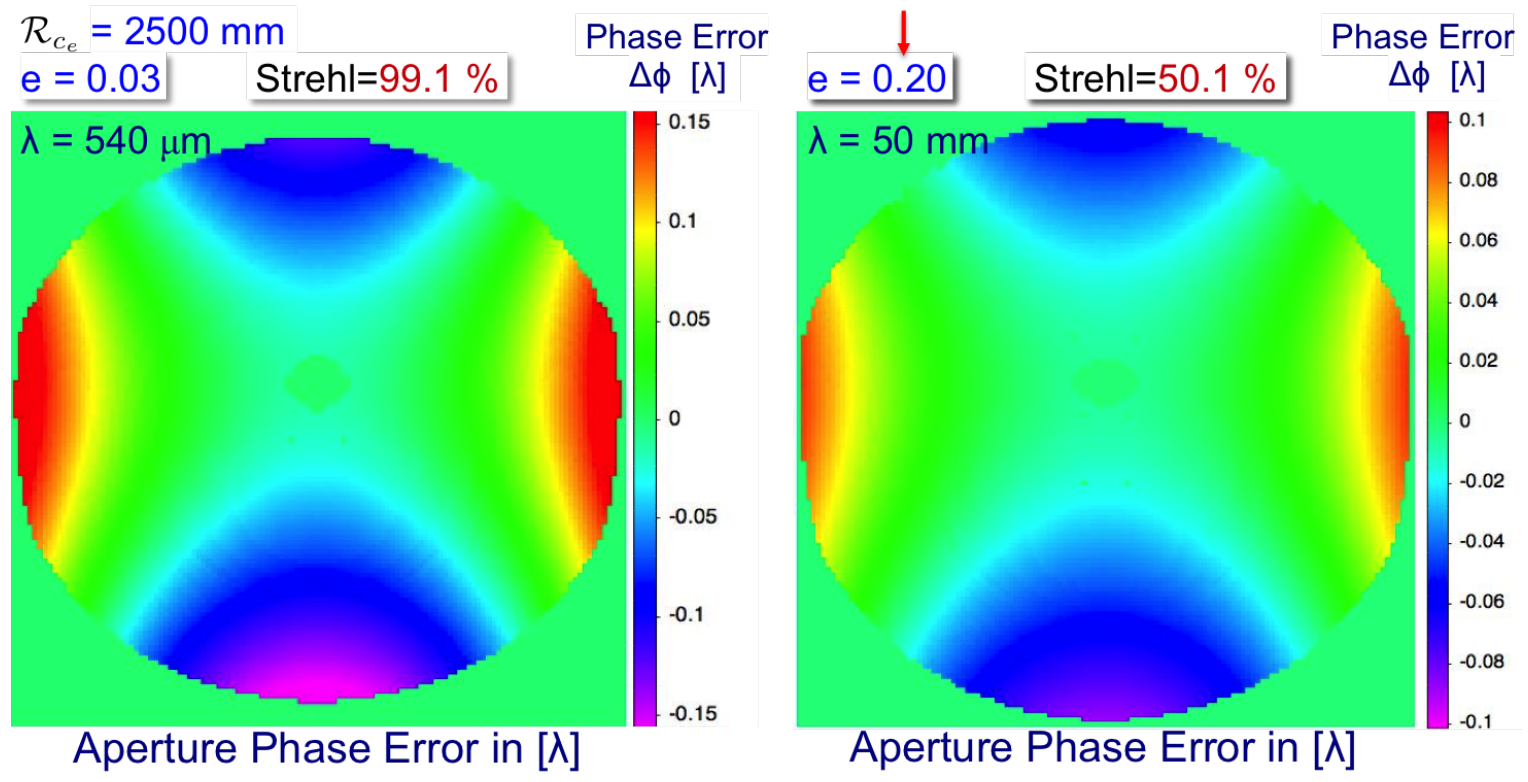

Figure 11. Aperture phase error distribution in wavelengths for different values of eccentricity for a $5 \mathrm{~m}$ balloon. Left: for an eccentricity $e=0.03$ and Strehl ratio of $99.1 \%$ at $540 \mu \mathrm{m}$, which corresponds to a foci separation of $0.15 \mathrm{~m}$. Right: eccentricity $e=0.2$, which corresponds to a foci separation of $1.05 \mathrm{~m}$.

\section{CONCLUSIONS}

LBR is a game-changing approach to realizing large aperture telescopes at THz frequencies (aka "the farinfrared"). In this paper we provide an optical prescription for LBR and examine its performance and tolerancing. We find that the design is capable of providing diffraction limited operation throughout the $\mathrm{THz}$ regime.

\section{ACKNOWLEDGMENTS}

This work was supported under the NASA Innovative Advanced Concepts (NIAC) grant NNX14AT48G. This work was done at the University of Antioquia, Medellin, Colombia, and in part at the Jet Propulsion Laboratory, which is operated for NASA by the California Institute of Technology.

\section{REFERENCES}

[1] C. Walker et al. "The Stratospheric THz Observatory (STO)", SPIE Proceedings Ground-based and Airborne Telescopes III, vol. 7733, No. 2, Jul. 2010.

[2] E. Pascale et al. "The Balloon-borne Large Aperture Submillimeter Telescope: BLAST", The Astropysical Journal, vol. 681, No. 1, Jan. 2008.

[3] A. Roy et al. "The Balloon-Borne Large Aperture Submillimeter Telescope (BLAST) 2005: A 10 Deg$^{2}$ Survey of Star Formation In Cygnus X", The Astrophysical Journal, vol. 727, No. 2, Jan. 2011.

[4] N. Galitzki. et al, "The Balloon-borne Large Aperture Submillimeter Telescope for Polarimetry-BLASTPol: Performance and results from the 2012 Antarctic flight", SPIE Proceedings on Astronomical Instrumentation, Ground-based and Airborne Telescopes V, 91450R, 22 July 2014.

[5] L. M. Fissel et al, "Balloon-Borne Submillimeter Polarimetry of the Vela C Molecular Cloud: Systematic Dependence of Polarization Fraction on Column Density and Local Polarization-Angle Dispersion", Accepted for publication in The Astrophysical Journal, 2016.

[6] P.-S. Kildal, L.A. Baker, Hagfors, T. "The Arecibo upgrading: electrical design and expected performance of the dual-reflector feed system". Proceedings of the IEEE, Vol. 82, Issue: 5, pp: 714-724, 1994. 